$\underline{\text { Supporting Information }}$

\title{
Carotenoid Nanovector for Efficient Therapeutic Gene Knockdown of Transcription Factor FOXC1 in Liver Cancer
}

Santosh K. Misra, ${ }^{a}$ Tania Ray, ${ }^{b}$ Fatemeh Ostadhossein, ${ }^{a}$ Bomy Kim, ${ }^{b}$ Partha S. Ray, ${ }^{b} *$ and

$$
\text { Dipanjan } \operatorname{Pan}^{a, *}
$$

${ }^{a}$ Department of Bioengineering and Beckman Institute, Materials Science and Engineering, University of Illinois at Urbana-Champaign and Carle Cancer Center, 502 N. Busey, Urbana, IL, 61801; E-mail:_dipanjan@illinois.edu

${ }^{b}$ Department of Surgery, University of Illinois College of Medicine, Division of Surgical Oncology, Carle Cancer Center, 509 W. University Avenue, Urbana, IL, 61801. E-mail: psray@illinois.edu 
Table of Content

S.N.

Content

Page No.

1. Synthesis of carotenoid-PAMAM

S3

2. ${ }^{1} \mathrm{H}$ NMR spectrum and the respective peak assignment of PAMAM dendrimer

S4

3. ${ }^{1} \mathrm{H}$ NMR spectrum of trans- $\beta$-Apo-8'-carotenal

S4

4. ${ }^{1} \mathrm{H}$ NMR spectrum of Carotenoid-dendrimer nanoparticles and the corresponding peak

assignment

S5

5. ${ }^{1} \mathrm{H}$ NMR peak assignment of trans- $\beta$-Apo-8'-carotenal

S5

6. Quantification of siRNA internalization efficiency from fluorescence imaging

S6 

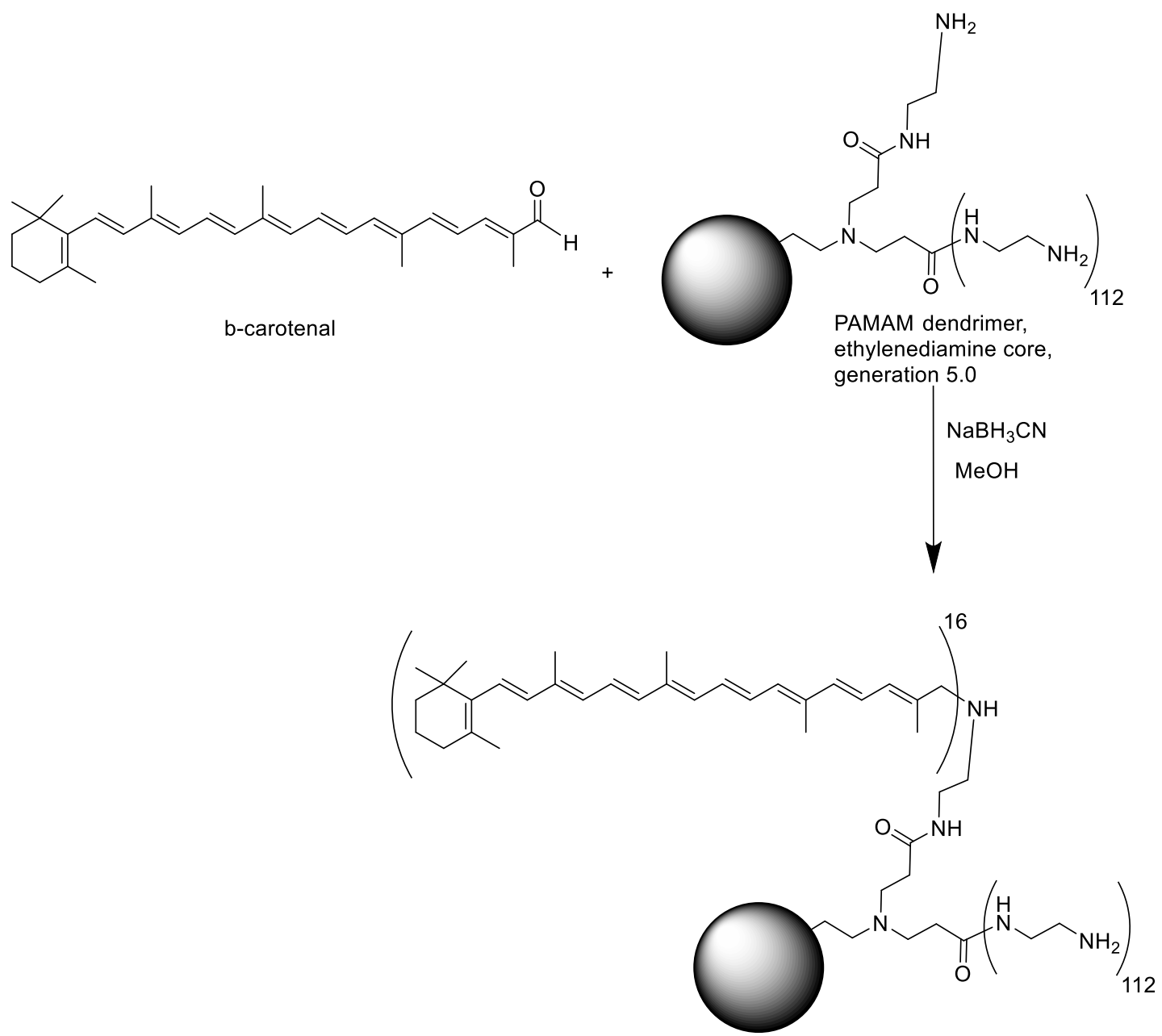

Molecular formula: $\mathrm{C}_{1744} \mathrm{H}_{3172} \mathrm{~N}_{506} \mathrm{O}_{252}$ $\mathrm{M} . \mathrm{W}=35,200 \mathrm{Da}$ (Nominal)

Total No of Carotene groups on the surface: 16

Scheme S1. Synthesis of carotenoid-PAMAM. 

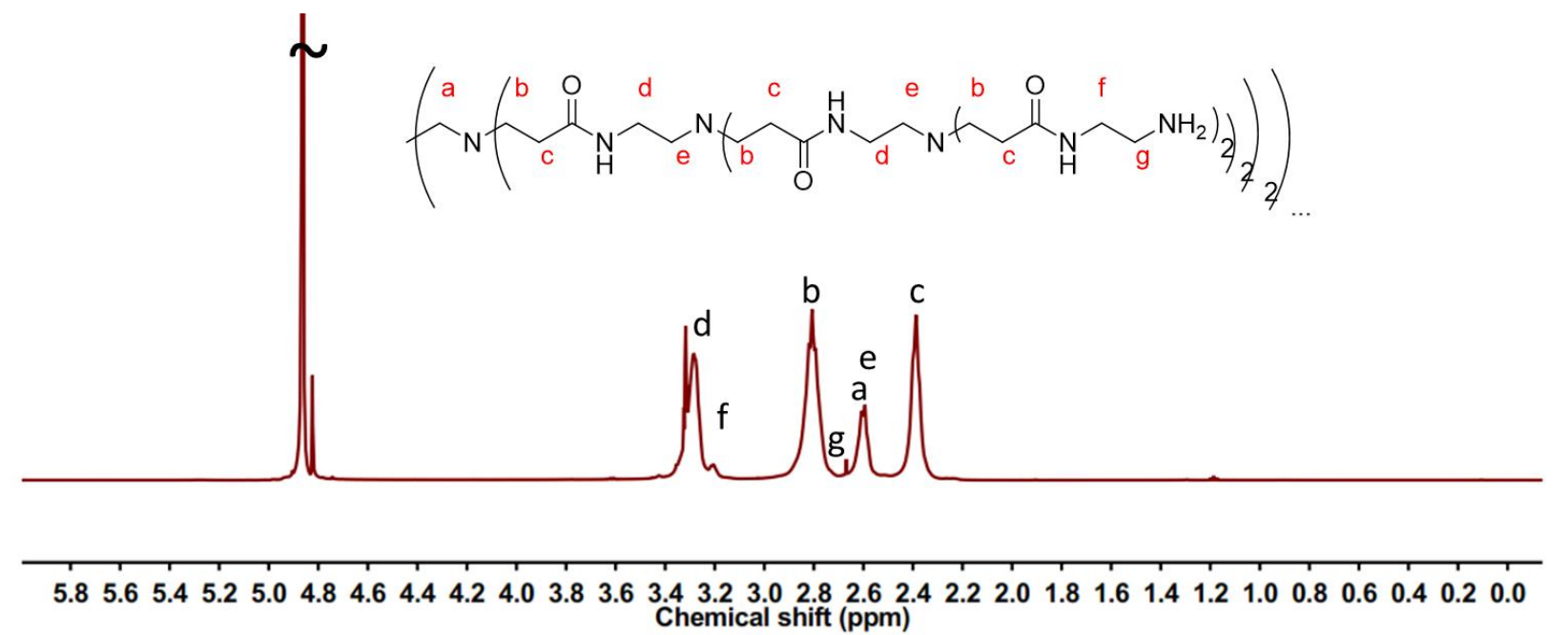

Figure S 1. The ${ }^{1} \mathrm{H}$ NMR spectrum and the respective peak assignment of PAMAM dendrimer.

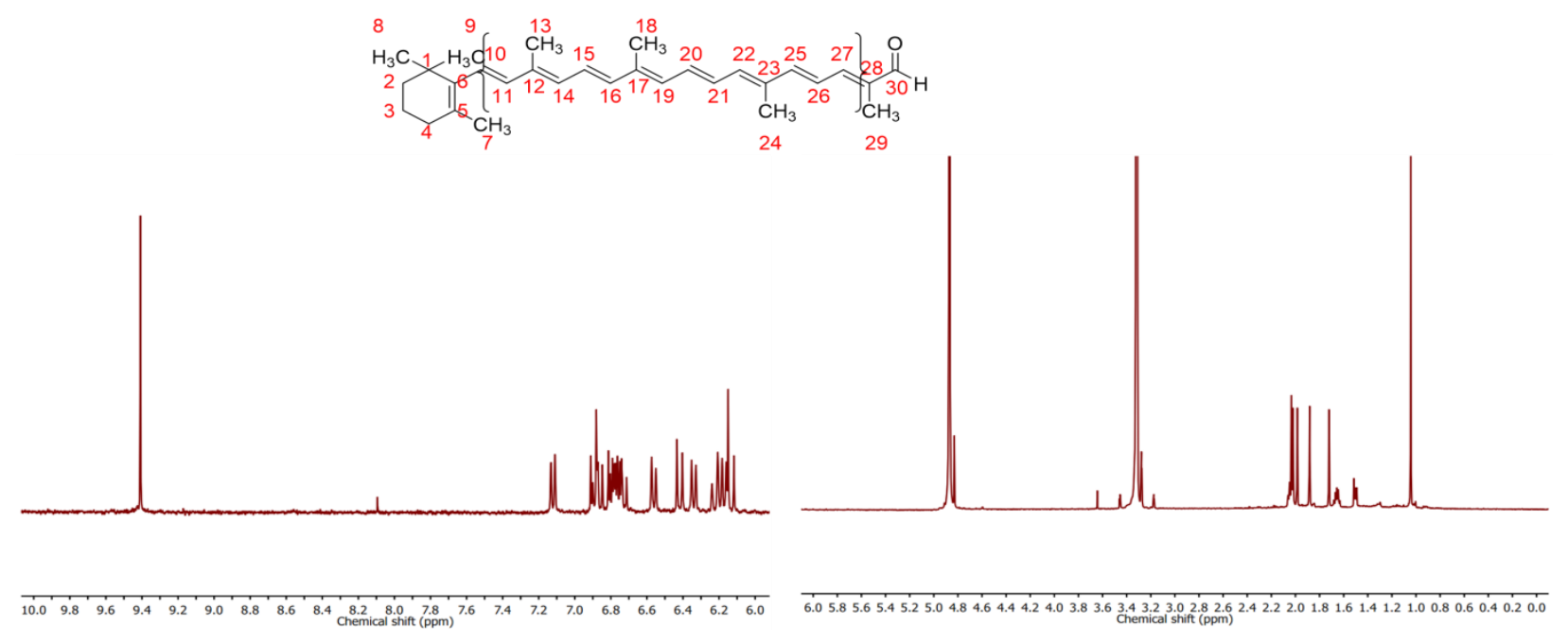

Figure S 2. The ${ }^{1} \mathrm{H}$ NMR spectrum of trans- $\beta$-Apo- 8 '-carotenal. The respective peak assignment is made in Table $\mathrm{S} 1$. 


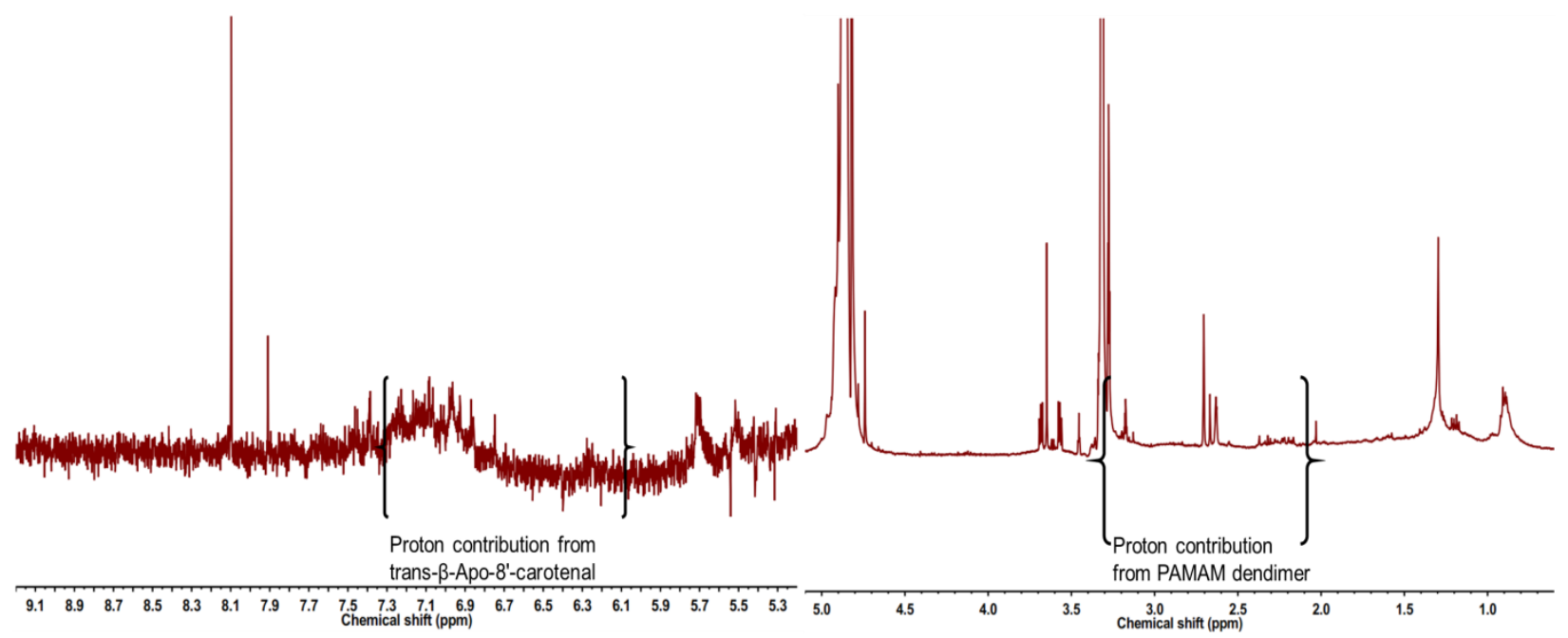

Figure S3. The ${ }^{1} \mathrm{H}$ NMR spectrum of Carotenoid-dendrimer nanoparticles and the corresponding peak assignment.

Table S1. The ${ }^{1} \mathrm{H}$ NMR peak assignment of trans- $\beta$-Apo- 8 '-carotenal.

\begin{tabular}{|c|c|c|c|}
\hline $\boldsymbol{\delta}(\mathbf{p p m})$ & Proton \# & $\boldsymbol{\delta}(\mathbf{p p m})$ & Proton \# \\
\hline $\mathbf{1 . 0 4}$ & 8,9 & $\mathbf{6 . 4 3}$ & 27 \\
\hline $\mathbf{1 . 4 9}$ & 2 & $\mathbf{6 . 5 7}$ & 11 \\
\hline $\mathbf{1 . 6 3}$ & 3 & $\mathbf{6 . 7 1}$ & 10 \\
\hline $\mathbf{1 . 7 2}$ & 24 & $\mathbf{6 . 7 4}$ & 19 \\
\hline $\mathbf{1 . 8 4}$ & 7 & $\mathbf{6 . 7 6}$ & 21 \\
\hline $\mathbf{1 . 8 8}$ & 18,29 & $\mathbf{6 . 8 0}$ & 20 \\
\hline $\mathbf{1 . 9 8}$ & 4,13 & $\mathbf{6 . 8 5}$ & 16 \\
\hline $\mathbf{6 . 1 5}$ & 15 & $\mathbf{6 . 9 0}$ & 24 \\
\hline $\mathbf{6 . 2 4}$ & 14 & $\mathbf{7 . 1 1}$ & 26 \\
\hline $\mathbf{6 . 3 5}$ & 22 & $\mathbf{9 . 4 1}$ & 30 \\
\hline
\end{tabular}



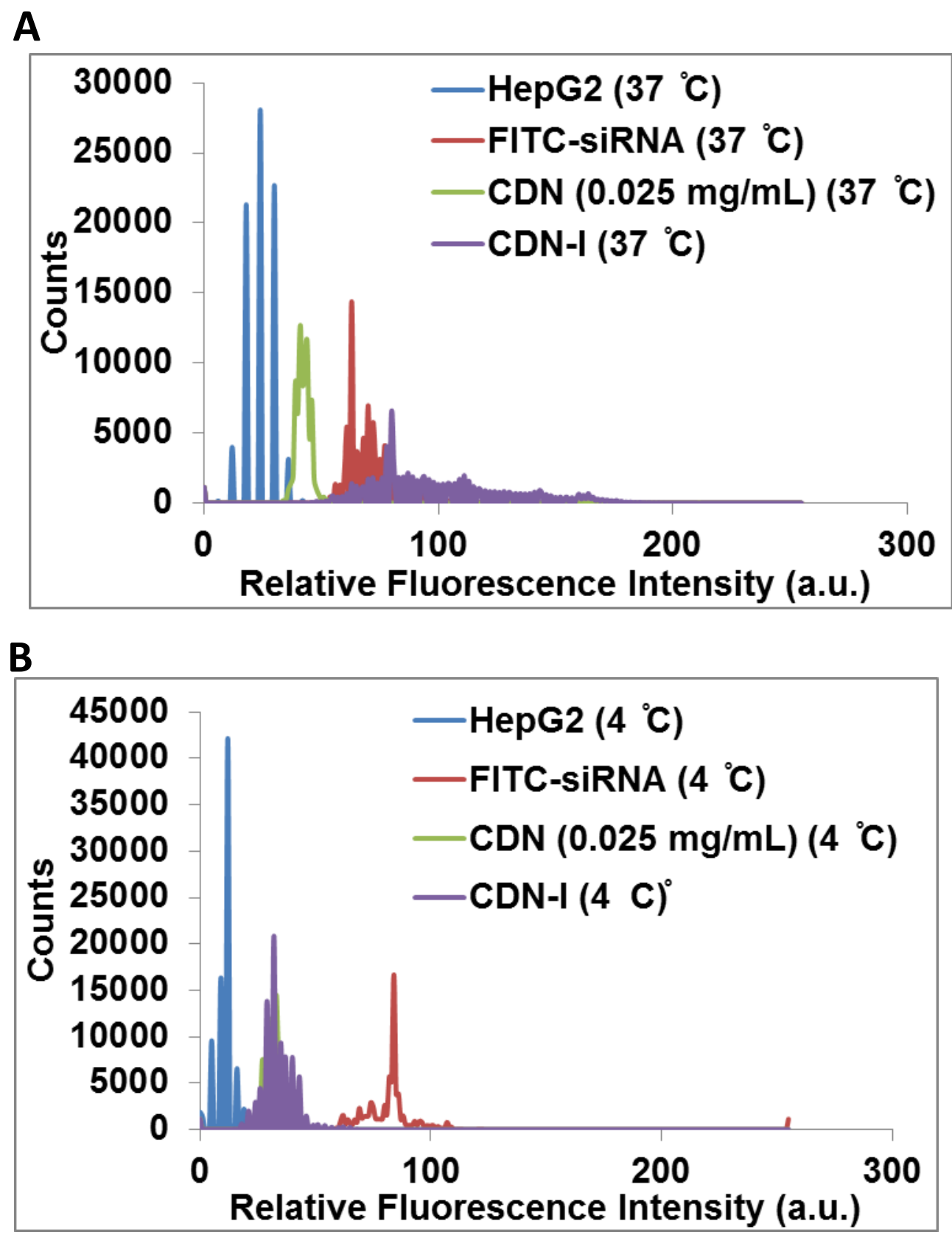

Figure S4. Quantification of siRNA internalization efficiency from fluorescence imaging at (A) $37^{\circ} \mathrm{C}$ and (B) $4^{\circ} \mathrm{C}$. Relative fluorescence intensity improved on $37^{\circ} \mathrm{C}$ temperature. Here CDN-I represents formulation NanosiFOXC1-I. 